

\title{
[Review] Bayram Balci, Renouveau de l'islam en Asie centrale et dans le Caucase
}

Enki Baptiste

\section{To cite this version:}

Enki Baptiste. [Review] Bayram Balci, Renouveau de l'islam en Asie centrale et dans le Caucase.

Lectures, 2018, 10.4000/lectures.24561 . hal-02551260

\section{HAL Id: hal-02551260 \\ https://hal.science/hal-02551260}

Submitted on 22 Apr 2020

HAL is a multi-disciplinary open access archive for the deposit and dissemination of scientific research documents, whether they are published or not. The documents may come from teaching and research institutions in France or abroad, or from public or private research centers.
L'archive ouverte pluridisciplinaire HAL, est destinée au dépôt et à la diffusion de documents scientifiques de niveau recherche, publiés ou non, émanant des établissements d'enseignement et de recherche français ou étrangers, des laboratoires publics ou privés. 
$\begin{array}{ll}\text { lectures } & \begin{array}{l}\text { Lectures } \\ \text { Les comptes rendus }\end{array}\end{array}$

\section{Bayram Balci, Renouveau de l'islam en Asie centrale et dans le Caucase}

\section{Enki Baptiste}

\section{(2) OpenEdition}

\section{Journals}

Édition électronique

URL : http://journals.openedition.org/lectures/24561

ISSN : 2116-5289

Éditeur

Centre Max Weber

Référence électronique

Enki Baptiste, "Bayram Balci, Renouveau de l'islam en Asie centrale et dans le Caucase », Lectures [En ligne], Les comptes rendus, 2018, mis en ligne le 11 avril 2018, consulté le 11 avril 2018. URL : http:// journals.openedition.org/lectures/24561

Ce document a été généré automatiquement le 11 avril 2018.

(c) Lectures - Toute reproduction interdite sans autorisation explicite de la rédaction / Any replication is submitted to the authorization of the editors 


\title{
Bayram Balci, Renouveau de l'islam en Asie centrale et dans le Caucase
}

\author{
Enki Baptiste
}

1 Cet ouvrage de Bayram Balci arrive dans un contexte de renouvellement de l'historiographie sur l'Asie centrale et le Caucase. Il apparaît comme l'une des rares publications de niveau universitaire sur la question, accessible en langue française et dont le contenu est abordable.

Olivier Roy, dans la préface, rappelle que l'Asie centrale suscite un intérêt croissant depuis la chute de l'URSS et la mise en place par la France d'un réseau diplomatique centrasiatique. Les premiers travaux universitaires ${ }^{1}$ attestent d'une résilience importante de l'islam, sous des formes spécifiques, malgré des années d'athéisme militant sous le régime soviétique et les politiques répressives contre les figures religieuses de la région. Bayram Balci se propose de faire un bilan de l'état du religieux et des formes de son renouveau en Asie centrale et dans le Caucase, une trentaine d'années après les indépendances. Le terrain de recherche comprend les républiques d'Asie centrale ${ }^{2}$ et l'Azerbaïdjan, périmètre qui se justifie par le fait que ces nouveaux États ont entrepris, à la chute de l'URSS, de se positionner vis-à-vis de l'islam et ont développé des politiques de gestion du religieux qui façonnent, jusqu'à aujourd'hui, l'islam centrasiatique et caucasien.

3 Pour traiter de la question du renouveau islamique, l'auteur entreprend une étude qui vise à déterminer quelles furent et quelles sont aujourd'hui les sources d'influence extérieures de ce renouveau. Postulant que, depuis la chute de l'URSS, la zone est entrée dans une globalisation du religieux, c'est-à-dire que ces pays importent ou subissent l'importation d'idées religieuses extérieures, Bayram Balci s'intéresse de plus près à quatre pays susceptibles d'influencer le renouveau islamique dans la région : la Turquie, l'Iran, l'Arabie saoudite et l'Inde. Suivant un plan académique facilitant grandement la lecture pour les non-initiés, l'auteur consacre un chapitre à des éléments de cadrage puis se penche, dans les quatre chapitres suivants, sur le rôle de ces quatre puissances extérieures. La dernière partie s'intéresse à la politique religieuse des États centrasiatiques et caucasiens. 
4 La réflexion s'amorce autour de l'évidente contradiction entre le fait que les États d'Asie centrale revendiquent fermement un héritage islamique et les politiques qu'ils mettent en place, encore très marquées par le sécularisme issu du soviétisme. Comme les travaux récents menés par des chercheurs du Cetobac ${ }^{3}$ l'ont montré ${ }^{4}$, le régime soviétique n'a jamais éliminé l'islam. Bien au contraire, certes reléguées dans la sphère privée, les pratiques religieuses se sont construites et ont évolué au gré des relations ambigües, par moment conflictuelles, entre les autorités soviétiques et les autorités religieuses de la région.

5 Le renouveau religieux, qui n'est d'ailleurs pas exclusivement musulman, remonte à l'arrivée au pouvoir de Gorbatchev. Les populations locales redécouvrent leur héritage islamique qu'elles cherchent à mettre en phase avec les nouvelles identités nationales, se réappropriant l'histoire, la remodelant et sanctifiant des personnages importants du passé local.

6 Les liens entre la Turquie, tout d'abord, et les nouvelles républiques d'Asie centrale sont surtout d'ordre linguistique ${ }^{5}$ et religieux ${ }^{6}$. Si, jusqu'en 1991, les relations entre la Turquie et les républiques soviétiques étaient inexistants, après l'implosion de l'URSS, les Turcs ont cherché à coopérer avec ces nouvelles républiques voisines, sans programme clair néanmoins. Outre les relations économiques, c'est dans le domaine culturel et religieux que la Turquie a joué et joue encore un rôle majeur d'influence en Asie centrale. Via la Diyanet, un organisme de gestion du religieux chargé d'administrer les lieux de culte, d'organiser les pèlerinages et l'éducation islamique, la Turquie participe au renouveau islamique centrasiatique, grâce à l'ouverture d'antennes dans les États et à la formation d'imams-prédicateurs. Ce sont aussi les entités non étatiques qui ont joué un rôle important dans l'expansion de l'influence turque. Depuis les réformes libérales initiées par Turgut Özal, dans les années 1980, un secteur privé dynamique et conservateur s'est développé dans le pays, dont les acteurs sont attirés par les activités missionnaires. Différents mouvements de prédication font ici l'objet d'une étude détaillée. L'auteur insiste sur le rôle important de la confrérie Nakshibendiyya, des disciples de Sait Nursi, mais surtout de la communauté du désormais bien connu Fethullah Gülen. Engagés dans l'ouverture d'écoles, les missionnaires gülenistes disposaient, jusqu'à fin 2013, du soutien du gouvernement turc. Ils ont participé à la mise en place d'un solide réseau de madrasas en Asie centrale. Après le 11 Septembre et la restriction de la présence des mouvements religieux d'origine étrangère dans les pays d'Asie centrale, les gülenistes ont adopté un mode de d'action nouveau, fondé sur l'exemplarité de leur comportement, agissant comme un soft power, et non plus une prédication religieuse assumée.

7 L'Iran, de son côté, est souvent présenté comme une puissance à l'action subversive chez ses voisins. Si l'héritage historique persan est indéniable en Asie centrale, les recherches de Bayram Balci montrent que la crainte que suscite l'Iran est un fantasme développé par les Occidentaux après la révolution islamique. L'intégralité du chapitre consacré à l'État perse s'attache à montrer à quel point la politique iranienne en Asie centrale est dominée par le pragmatisme géopolitique et non par des velléités d'expansion religieuse. Lors de la guerre du haut Karabagh (1988-1994), Téhéran, afin de briser l'axe Bakou-Ankara, n'a pas soutenu explicitement l'Azerbaïdjan, pourtant puissance chiite du Caucase.

8 En marge des tractations géopolitiques sur les hydrocarbures disputés de la mer Caspienne, l'Iran a raté le coche sur la scène centrasiatique. Lorsque l'URSS a éclaté, après des années d'un système dirigiste, les républiques d'Asie centrale ont cherché à se développer selon un modèle économique libéral, incarné alors par les États-Unis. Le 
régime islamique iranien de l'époque effrayait quant à lui les élites centrasiatiques, très marquées par le sécularisme soviétique. Sur le plan religieux, l'Iran tente de mettre en place des politiques de coopération culturelle avec les pays d'Asie centrale abritant des minorités chiites. Cela concerne les Ironi d'Ouzbékistan et du Turkménistan, les Azéris d'Azerbaïdjan et les Ismaéliens du Badakhshan. Mais les dissensions idéologiques et les allégeances religieuses variables rendent l'emprise de l'Iran sur ces minorités très tenue. En Asie centrale et en Azerbaïdjan, l"accusation d'être un espion de l'Iran est devenue un moyen aisé de discréditer les mouvements religieux et politiques contestataires.

Les deux derniers chapitres sont consacrés aux influences saoudiennes et indiennes dans l'espace centrasiatique. Bayram Balci opte pour une approche du rôle saoudien en Asie centrale à travers la gestion du pèlerinage (hadj) et de l'émigration turkestanaise en péninsule Arabique. Avant la fermeture soviétique, la Russie des Tsars avait mis en place une politique de gestion du hadj à travers une série de point-relais pour les fidèles musulmans dans les villes ottomanes. Après la chute de l'URSS, les musulmans ont réinvesti les circuits du hadj, reconnectant les espaces arabes et post-soviétiques. Terrifiés par la propagation de l'islam wahhabite, les régimes s'efforcent néanmoins de limiter la circulation des idées. L'influence de l'Arabie Saoudite passe aussi par la gestion d'une émigration centrasiatique importante datant de l'époque soviétique, qui concerne les communautés ouïghours et ouzbeks. La lecture de cette partie laisse cependant le lecteur sur sa faim. La maigreur de l'historiographie sur le sujet aurait justifié que l'auteur s'attarde plus amplement sur les modalités de gestion et les circonstances de l'implantation de ces communautés en péninsule Arabique. On apprend néanmoins que, depuis l'effondrement du bloc soviétique, le lien entre ces émigrés et leurs régions d'origine s'étiole à mesure de l'arabisation des jeunes nés en Arabie Saoudite.

Comme l'Iran, l'Arabie Saoudite pâtit de son image de promoteur de l'islamisme. Cela a entamé sa réputation et a conduit les républiques d'Asie centrale à limiter la circulation des idées entre leur territoire et le monde arabe.

11 L'Asie du sud partage une histoire ancienne avec l'Asie centrale, mais leurs liens religieux sont surtout le fait récent d'une confrérie, la Jama'at al-Tabligh, émanation de l'école sunnite deobandi. En Asie centrale, elle a suscité beaucoup de méfiance, car son enseignement est basé sur la pratique du tabligh, une prédication qui vise à ramener les musulmans vers une pratique épurée de la foi Le seul pays où elle est parvenue à s'implanter est le Kirghizstan, où l'État a fait le choix d'instrumentaliser la confrérie pour encadrer une jeunesse désœuvrée et susceptible de céder aux discours islamistes.

12 Auparavant renvoyées dans la sphère privée, après la chute de l'URSS, les pratiques religieuses ont retrouvé une place publique et la religion a été «déprivatisée ». L'Asie centrale a connu un intense renouveau religieux. La gestion spécifique du développement de l'islam dans les pays concernés justifie de parler des islams d'Asie centrale, tant la diversité de leurs formes est importante. L'attitude des États est ambigüe et, si le sécularisme soviétique a laissé des traces, ils font montre d'un interventionnisme croissant dans les affaires religieuses, obsédés par la pénétration d'idées corrosives et par le risque terroriste. L'auteur estime ainsi que «la muséification des établissements religieux [...] cristallise l'islam dans des décors délimités/définis, plus qu'elle ne sert l'épanouissement de la pratique » (p. 260).

13 L'ouvrage a le mérite de traiter de manière pédagogique et synthétique des enjeux religieux dans un espace immense et méconnu du grand public. L'auteur minimise - trop, selon nous - le défi djihadiste et le risque islamiste dans les pays d'Asie centrale. On 
regrettera l'absence d'étude sur les régions russes du Caucase, notamment la Tchétchénie, le Daghestan et l'Ingouchie, particulièrement touchées par les discours radicaux. Sur le plan formel, il est regrettable que le manuscrit n'ait pas fait l'objet d'une relecture attentive. Le texte est en effet émaillé de nombreuses fautes d'orthographe et imprécisions langagières.

Ce voyage en Asie centrale permet au lecteur d'appréhender la région comme un véritable laboratoire ${ }^{7}$ de refondation du religieux. Comme le souligne l'auteur, les républiques centrasiatiques, en construisant ex nihilo de nouvelles identités nationales, ferment la parenthèse soviétique. L'islam joue un rôle de premier plan pour ces sociétés qui, dans un contexte de globalisation du religieux, s'abreuvent désormais à des sources d'influence qui ne cessent de se multiplier.

\section{NOTES}

1. Voir notamment les travaux d'Alexandre Bennigsen, parmi lesquels: Muslims in the Soviet Empire, a Guide, Londres, C. Hurst, 1985 ; « Die Islamische Zeitkombe in Mittelasien », Weltmacht Islam, $\mathrm{n}^{\circ} 1,1988$, pagination inconnue.

2. Le Kazakhstan, le Kirghizstan, l'Ouzbékistan, le Tadjikistan et le Turkménistan.

3. Centre d'études turques, balkaniques et centrasiatiques, UMR 8033, EHESS.

4. Voir en particulier les travaux sur l'islam soviétique centrasiatique de Stéphane Dudoignon, notamment, Dudoignon Stéphane, Christian Noack (dir.), Allah's Kolkhozes. Migration, DeStalinisation, Privatisation and the New Muslim Congregations in the Soviet Realm (1950s-2000s), Berlin, Klaus Schwarz Verlag, 2014.

5. Les langues d'Asie centrale et d'Azerbaïdjan sont dites turciques, à l'exception de celles du Tadjikistan.

6. Les pays de la région sont de rite sunnite hanafite, comme la Turquie, sauf l'Azerbaïdjan et le Tadjikistan, qui sont majoritairement chiites.

7. Le terme est de nous.

\section{AUTEUR}

\section{ENKI BAPTISTE}

Médiéviste et arabisant à l'Université Lumière Lyon 2 (Ciham, UMR 5648), travaille sur les débuts de l'islam au Moyen-Orient, notamment à travers l'historiographie et la question des représentations de l'institution califale. 